\title{
Response of timothy to increasing rates of potassium
}

\author{
INTO SAARELA
}

\author{
Department of Agricultural Chemistry and Physics, Agricultural Research \\ Centre, 31600 Jokioinen
}

\begin{abstract}
Five potassium fertilization rates ranging from nil to $80 \mathrm{~kg} / \mathrm{ha} /$ cut were compared over 2 to 3 years in field trials on timothy leys at nine sites between 61 and $65^{\circ} \mathrm{N}$. The grass was cut twice a year and the contents of nitrogen, potassium, calcium and magnesium in yields were determined. The soils were tested at the beginning and at the end of the trials.

On four peat soils the yields over two years without potassium dressings were 34 to $66 \%$ of the respective yields with adequate potassium fertilization. On humus soil the relative yield without potassium was $81 \%$ and on finesand soil $76 \%$. On two finesand tills rich in organic matter the responce of timothy to potassium was $5 \%$. No significant yield response was obtained on silty clay.

In accordance to the depletion of available soil reserves, the differences between the potassium rates increased with time. In average on the six most responsive soils the relative yields without potassium fertilization for the first four successive cuts were $88,75,58$ and $45 \%$. For maximum yields, 60 to $80 \mathrm{~kg} /$ ha potassium per cut was required on the organogenic soils and on the finesand, $20 \mathrm{~kg} / \mathrm{ha}$ was enough on the other three mineral soils.

The potassium contents of plants increased greatly, and the contents of nitrogen, calcium and magnesium decreased with increasing potassium fertilization rate. The magnesium content of grass rose to an unusually high level with severe potassium deficiencies. At the end of the trials the soils were quite exhausted of potassium, the subsurface layers being most exhausted.

The critical plant potassium content varied from under $2 \%$ to over $3 \%$. As the large variation was coupled with plant nitrogen, plant $\mathrm{K} / \mathrm{N}$ ratio was a better indicator for potassium status of ley than plant $\mathrm{K}$. Yield was likely to begin degreasing when the $\mathrm{K} / \mathrm{N}$ ratio decreased under 1 .
\end{abstract}

\section{Introduction}

The requirement of potassium fertilization of ley crops on finnish soils has been investigated by means of a number of field experiments. Potassium application has been essential for good yields on organogenic soils and on coarse mineral soils (SALONEN and TAINIO 1961), but silt and clay soils have usually produced maximum yields without any added potassium (KERÄNEN and TAINIO 1968).

Since these and other older experiments have been carried out, a much more intensive cropping of leys has become a general practice. The manipulation of the growth of grasses by heavy nitrogen dressings, which is a key to high energy and protein yields (HIIVOLA et al. 1974), greatly increases 
potassium uptake and hastens depletion of potassium reserves of soil (JOY et al. 1973, SILLANPÄÄ and RINNE 1975, TÄHTINEN 1979).

The increased uptake may be compensated by increased amounts of applied potassium, but too heavy a potassium dressing is harmful, as excess potassium changes the mineral composition of plants poorer in animal nutrition (ETTALA and KOSSILA 1979). To maintain the potassium of leys within the sufficient but not excessive range, fertilizer potassium should be applied in frequent small dressings. Excluding clayey soils having a high potassium buffer power, a separate dressing for every cut is preferable (MELA et al. 1977, PELTOMAA et al. 1979, SAARELA et al. 1981).

The aim of this study is to investigate how large amounts of fertilizer potassium should be applied on Finnish soils in intensive ley cropping. An estimation of the requirement of potassium fertilization by means of soil and plant tests is also examined.

\section{Material and methods}

The material comprises nine 2 to 3 year field trials on timothy leys cut twice a year. The treatments in comparison are five potassium fertilization rates from nil to eighty kilograms potassium a hectar with equal differences of twenty kilograms between the rates. Potassium was applied as potassium chloride fertilizer $(50 \% \mathrm{~K})$ separately for every cut:

Single dressisng
$0 \mathrm{~kg} \mathrm{~K} / \mathrm{ha}$
$20 "$ "
$40 \quad "$
$60 \quad "$
$80 \quad "$

Total in a year
$0 \mathrm{~kg} \mathrm{~K} / \mathrm{ha}$
40 "
80 "
120 "
160 "

Potassium was topdressed at the beginning of the growing seasons and immediately after the first cut. At the same times, ammonium nitrate limestone $(27.5 \% \mathrm{~N})$ was dressed at the rate of $80 \mathrm{~kg} \mathrm{~N} / \mathrm{ha}$. Superphosphate $(8.7 \% \mathrm{P})$ was dressed at the beginning of the growing seasons (45 kg P/ha).

A randomized block desing with four replicates, modified to limit the differences between adjacent plots to $40 \mathrm{~kg} \mathrm{~K} /$ cut, was used in the trials. The gross areas of the plots were $50 \mathrm{~m}^{2}$ and the harvested and weighed areas were 10-16 $\mathrm{m}^{2}$. Trials 1 to 5 were started in 1977, trials 6 to 9 in 1978 .

The soils were sampled before the first spreading of fertilizers and at the end of the trials. The soil $\mathrm{pH}_{(\mathrm{H} 2 \mathrm{O})}$ and the nutrients extractable into acid ammonium acetate (x-AAA) were determined according to VUORINEN and MÄKITIE (1955) as described also by TARES and SIPPOLA (1978). The particlesize distribution was analysed using ELONEN's (1971) pipette method. The organic carbon was determined using a colorimetric dichromate combustion method (TARES and SIPPOLA 1978). The HCL-extractable potassium (KHCL) was extracted into hot $2 \mathrm{M} \mathrm{HCL}$ (EGNER et al. 1960) in a 1:25 volume ratio. Data on soil properties at the beginning of the trials is given in Table 1. 
Table 1. Properties of soils at the trial sites.

\begin{tabular}{|c|c|c|c|c|c|c|c|c|c|c|c|c|c|c|c|}
\hline \multirow[t]{2}{*}{ Trial } & \multirow[t]{2}{*}{ Location } & \multirow[t]{2}{*}{ Depth } & \multirow[t]{2}{*}{ Soil type } & \multicolumn{6}{|c|}{ Org. $\mathrm{C}^{1)}$ Particle-size distr. $(\mathrm{mm})^{1)}$} & \multirow{2}{*}{$\begin{array}{l}\mathrm{pH}^{2)} \\
\left(\mathrm{H}_{2} \mathrm{O}\right)\end{array}$} & \multicolumn{5}{|c|}{$\mathrm{mg} / 1^{2)}$} \\
\hline & & & & - & .002 & .02 & .2 & 2.0 & & & $\mathrm{Ca}$ & $\mathrm{Mg}$ & $P$ & $\mathrm{~K}_{\mathrm{AAA}}$ & $\mathrm{K}_{\mathrm{HCL}}$ \\
\hline \multirow[t]{2}{*}{1} & Hartola & $0-20$ & Finesandy till & 7.0 & 14 & 34 & 27 & 20 & 5 & 5.4 & 945 & 43 & 7.0 & 102 & 1648 \\
\hline & $61^{\circ} 30^{\prime} \mathrm{N}$ & $20-40$ & Finesandy till & 0.3 & 16 & 38 & 30 & 15 & 1 & 5.7 & 342 & 64 & 1.4 & 86 & 3235 \\
\hline \multirow[t]{2}{*}{2} & Hartola & $0-20$ & Finesandy till & 7.5 & 16 & 36 & 27 & 17 & 4 & 5.4 & 1065 & 55 & 8.4 & 149 & 1910 \\
\hline & $61^{\circ} 30^{\prime} \mathrm{N}$ & $20-40$ & Finesandy till & 0.3 & 13 & 35 & 31 & 20 & 1 & 5.5 & 280 & 52 & 2.0 & 92 & 3797 \\
\hline \multirow[t]{2}{*}{3} & Ilomantsi & $0-20$ & Ligno Carex peat & 39 & - & - & - & - & - & 5.2 & 3260 & 138 & 11.4 & 73 & 148 \\
\hline & $63^{\circ} \mathrm{N}$ & $20-40$ & Ligno Carex peat & 45 & - & - & - & - & - & 5.2 & 2905 & 190 & 4.4 & 50 & 204 \\
\hline \multirow[t]{2}{*}{4} & Pihtipudas & $0-20$ & Carex peat & 38 & - & - & - & - & - & 5.8 & 2002 & 440 & 2.6 & 34 & 74 \\
\hline & $63^{\circ} 30^{\prime} \mathrm{N}$ & $20-40$ & Carex peat & 40 & - & - & - & - & - & 5.5 & 1700 & 417 & 1.8 & 28 & 64 \\
\hline \multirow[t]{2}{*}{5} & Muhos & $0-20$ & Finesand & 4.2 & 3 & 2 & 7 & 81 & 7 & 6.3 & 812 & 173 & 7.0 & 86 & 158 \\
\hline & $65^{\circ} \mathrm{N}$ & $20-40$ & Finesand & 0.9 & 1 & 1 & 7 & 87 & 4 & 5.6 & 128 & 45 & 1.1 & 18 & 122 \\
\hline \multirow[t]{2}{*}{6} & Pihtipudas & $0-20$ & Humus soil & 13 & - & - & - & - & - & 6.0 & 1640 & 214 & 4.2 & 46 & 199 \\
\hline & $63^{\circ} 30^{\prime} \mathrm{N}$ & $20-40$ & Gyttja & 7.0 & 47 & 42 & 10 & 1 & 0 & 5.8 & 810 & 158 & 0.9 & 51 & 350 \\
\hline \multirow[t]{2}{*}{7} & Pihtipudas & $0-20$ & Silty clay & 3.5 & 33 & 48 & 18 & 1 & 0 & 5.4 & 1030 & 321 & 3.1 & 102 & 1960 \\
\hline & $63^{\circ} 30^{\prime} \mathrm{N}$ & $20-40$ & Carex peat & 27 & - & - & - & - & - & 5.2 & 1060 & 174 & 2.4 & 49 & 604 \\
\hline \multirow[t]{2}{*}{8} & Tyrnävä & $0-20$ & Carex peat & 24 & - & - & - & - & - & 5.0 & 1230 & 383 & 7.1 & 49 & 95 \\
\hline & $65^{\circ} \mathrm{N}$ & $20-40$ & Finesand & 4.4 & 1 & 1 & 3 & 93 & 2 & 4.2 & 138 & 37 & 4.2 & 22 & 157 \\
\hline \multirow[t]{2}{*}{9} & Utajärvi & $0-20$ & Carex peat & 35 & - & - & - & - & - & 5.0 & 1670 & 49 & 3.9 & 44 & 70 \\
\hline & $65^{\circ} \mathrm{N}$ & $20-40$ & Carex peat & 42 & - & - & - & - & - & 5.0 & 2260 & 109 & 1.0 & 23 & 40 \\
\hline
\end{tabular}

1) $=$ means of 4 samples, 2) $=$ means of 20 samples

The yields were weighed and sampled immediately after cuts. The percentages of air dry matter in the fresh samples were determined and the dry matter yields were calculated estimating the air dry moisture to be $15 \%$. The contents of potassium, calcium and magnesium in plant samples were determined according to KÄHÄRI and NISSINEN (1978). Total plant nitrogen was determined by means of the Kjeldahl-procedure using a Kjeltec-apparatus (Tecator, Sweden).

The differences between the means of the potassium fertilization rates were tested by Duncan's multiple range test. Values that do not differ significantly $(0.05)$ are indicated by the same letters. Dependences of the dry matter yields on soil and plant variables were calculated using stepwise regression analyses.

\section{Results and discussion}

Dry matter yields

Potassium fertilization increased dry matter yields (Table 2) significantly on all but one of the nine sites. In the first cuts the response was significant at two sites only, but the differences between the treatments increased with 
Table 2. Dry matter yields of timothy leys with increasing potassium fertilization rates $(\mathrm{kg} / \mathrm{ha})$.

\begin{tabular}{|c|c|c|c|c|c|c|c|c|c|}
\hline \multirow[t]{2}{*}{$\mathrm{K}$ rate, $\mathrm{kg} / \mathrm{ha}$} & \multicolumn{9}{|c|}{ Trial } \\
\hline & 1 & 2 & 3 & 4 & 5 & 6 & 7 & 8 & 9 \\
\hline & \multicolumn{9}{|c|}{1 st year 1 st cut } \\
\hline 0 & $5880^{4}$ & $4530^{2}$ & $3130^{4}$ & $4420^{a}$ & $4470^{2}$ & $4230^{a}$ & $4050^{a}$ & $1490^{a}$ & $3690^{4}$ \\
\hline 20 & $6070^{a}$ & $4780^{4}$ & $2970^{a}$ & $4670^{2}$ & $3980^{2}$ & $4340^{2}$ & $4130^{4}$ & $1580^{a}$ & $4350^{2}$ \\
\hline 40 & $5650^{2}$ & $4990^{4}$ & $3330^{a}$ & $4990^{a b}$ & $4390^{2}$ & $4290^{a}$ & $4220^{4}$ & $1680^{a}$ & $4690^{\mathrm{ab}}$ \\
\hline 60 & $5280^{4}$ & $4400^{4}$ & $3590^{4}$ & $5350^{b}$ & $4520^{1}$ & $4640^{a}$ & $4080^{4}$ & $1740^{2}$ & $5000^{b}$ \\
\hline \multirow[t]{2}{*}{80} & $5790^{4}$ & $4410^{4}$ & $3020^{a}$ & $5410^{b}$ & $4250^{4}$ & $4580^{2}$ & $3830^{4}$ & $1810^{a}$ & $5210^{4}$ \\
\hline & \multicolumn{9}{|c|}{1 st year 2 nd cut } \\
\hline 0 & $5970^{2}$ & $5750^{a}$ & 5180 & $2100^{2}$ & $1610^{4}$ & $3550^{a}$ & $4780^{a}$ & 4480 & 1020 \\
\hline 20 & $5420^{a}$ & $5960^{a}$ & $6670^{a}$ & $2590^{\mathrm{ab}}$ & $1710^{a}$ & $3630^{a}$ & $5300^{a}$ & $5500^{a}$ & 1640 \\
\hline 40 & $5340^{x}$ & $5350^{a}$ & $6430^{4}$ & $2920^{\mathrm{bc}}$ & $1760^{a}$ & $4110^{a}$ & $5080^{2}$ & $6010^{2}$ & 2090 \\
\hline 60 & $5740^{a}$ & $5940^{4}$ & $6790^{a}$ & $3340^{\circ}$ & $1700^{4}$ & $3940^{b}$ & $4640^{a}$ & $5520^{a}$ & $2350^{2}$ \\
\hline \multirow[t]{2}{*}{80} & $6610^{4}$ & $5800^{4}$ & $6810^{a}$ & $3240^{\circ}$ & $1820^{a}$ & $4300^{b}$ & $4990^{a}$ & $5300^{a}$ & $2460^{a}$ \\
\hline & \multicolumn{9}{|c|}{2 nd year 1 st cut } \\
\hline 0 & $7020^{\circ}$ & $7400^{2}$ & 2530 & 2000 & 4960 & $2540^{a}$ & $3020^{4}$ & 1170 & 1500 \\
\hline 20 & $7550^{4}$ & $7620^{\mathrm{ab}}$ & $3470^{2}$ & $3450^{2}$ & $6360^{4}$ & $2630^{a}$ & $3230^{4}$ & 1570 & 2990 \\
\hline 40 & $7590^{4}$ & $8030^{b}$ & $3920^{4}$ & $3890^{\text {ab }}$ & $6980^{1}$ & $2780^{4}$ & $3180^{4}$ & $1770^{2}$ & $4440^{2}$ \\
\hline 60 & $7510^{4}$ & $7620^{a b}$ & $4280^{a}$ & $4280^{b}$ & $7130^{4}$ & $3350^{b}$ & $3180^{2}$ & $1770^{2}$ & $4310^{2}$ \\
\hline \multirow[t]{2}{*}{80} & $7750^{2}$ & $7340^{2}$ & $3880^{2}$ & $4510^{b}$ & $6890^{\circ}$ & $3220^{\mathrm{b}}$ & $3080^{4}$ & $1930^{a}$ & $4740^{4}$ \\
\hline & \multicolumn{9}{|c|}{2 nd year 2 nd cut } \\
\hline 0 & $5750^{a}$ & $5690^{4}$ & 3060 & 1960 & 1390 & $3030^{2}$ & $3920^{4}$ & 1330 & 370 \\
\hline 20 & $6240^{4}$ & $5980^{2}$ & 4590 & 2930 & 2320 & $3670^{a b}$ & $4230^{4}$ & 2620 & 1500 \\
\hline 40 & $5880^{x}$ & $6110^{2}$ & $5410^{a}$ & $4190^{4}$ & $2910^{4}$ & $4020^{a b}$ & $4050^{2}$ & $3270^{2}$ & 2410 \\
\hline 60 & $5940^{2}$ & $5720^{4}$ & $5430^{2}$ & $4350^{2}$ & $3240^{a}$ & $3960^{\mathrm{ab}}$ & $4300^{a}$ & $3670^{a b}$ & 2980 \\
\hline \multirow[t]{2}{*}{80} & $5880^{2}$ & $5510^{2}$ & $5680^{2}$ & $3940^{2}$ & $3390^{2}$ & $4340^{b}$ & $4330^{4}$ & $3900^{b}$ & 3550 \\
\hline & \multicolumn{9}{|c|}{ Average in 1 st and 2 nd year } \\
\hline 0 & $12310^{a}$ & $11690^{a b}$ & 3420 & 5250 & 6210 & $6680^{a}$ & $7880^{4}$ & 4240 & 3300 \\
\hline 40 & $12640^{a}$ & $12170^{b}$ & 8850 & 6830 & $7200^{2}$ & $7110^{a b}$ & $8440^{4}$ & 5640 & 5250 \\
\hline 80 & $12230^{4}$ & $12240^{b}$ & $9550^{4}$ & $8000^{2}$ & $7990^{\mathrm{ab}}$ & $7620^{\mathrm{bc}}$ & $8280^{2}$ & $6360^{a}$ & 6830 \\
\hline 120 & $12240^{2}$ & $11840^{\mathrm{ab}}$ & $10050^{2}$ & $8660^{2}$ & $8180^{b}$ & $7950^{c}$ & $8120^{4}$ & $6350^{2}$ & 7320 \\
\hline \multirow[t]{4}{*}{160} & $13020^{2}$ & $11530^{2}$ & $9700^{2}$ & $8530^{2}$ & $8170^{b}$ & $8210^{\circ}$ & $8100^{4}$ & $6470^{a}$ & 7970 \\
\hline & - & * & 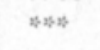 & क⿻ & 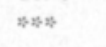 & 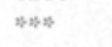 & - & $w n$ & 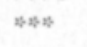 \\
\hline & \multicolumn{4}{|c|}{3 rd year trial 3} & \multicolumn{5}{|c|}{ 3rd year trial 5} \\
\hline & 1 st cut & 2nd cut & & & 1 st cut & 2nd cut & & & \\
\hline 0 & 3230 & 2110 & & & 1500 & 1040 & & & \\
\hline 20 & $4720^{a}$ & $5100^{\alpha}$ & & & 2780 & $2240^{a}$ & & & \\
\hline 40 & $5450^{a b}$ & $5920^{a}$ & & & $3310^{2}$ & $2390^{4}$ & & & \\
\hline 60 & $5670^{\mathrm{ab}}$ & $7050^{b}$ & & & $3690^{2}$ & $2780^{b}$ & & & \\
\hline 80 & $6080^{\mathrm{b}}$ & $8140^{\mathrm{b}}$ & & & $3510^{a}$ & $2810^{\mathrm{b}}$ & & & \\
\hline
\end{tabular}

time in accordance with the depletion of available soil potassium reserves. On average on the six most responsive soils, the omission of potassium fertilization caused in the first four successive cuts yield decreases of 12, 25, 42 and 55 $\%$. On the four peat soils the yields over two years were without potassium fertilization 34 to $66 \%$, on the humus soil $81 \%$ and on the finesand soil 76 $\%$ of the maximum yield obtained with adequate potassium dressings. On 
the other three mineral soils the response of timothy to potassium was no more than $5 \%$.

For maximum yields, 60 to $80 \mathrm{~kg}$ potassium per cut was required on the organogenic soils and on the finesand, $20 \mathrm{~kg}$ potassium per cut was enough on the other three mineral soils. The required amount of potassium increased with time. In the first cut no significant differences in yield were found between the four amounts of potassium fertilizer. In the last cuts on the peat soils the yields tended to be highest with the highest rate, the difference between 60 and $80 \mathrm{~kg} \mathrm{~K}$ being significant at one site.

\section{Nutrient contens}

Plant potassium content (Table 3 ) was extremely variable, the lowest being $6.4 \mathrm{~g} / \mathrm{kg}$ and the highest $45.8 \mathrm{~g} / \mathrm{kg}$. The differences between the soils were largest without potassium, as potassium fertilization increased the contents most on soils with the lowest amounts of available potassium. The large variability between cuts in some trials was due to different stages of development, as the plant potassium content decreases with advancing maturity.

Plant potassium contents were not systematically different in the first and second cuts of a year. The same result has also been obtained in other studies when the potassium had been applied separately for every cut in equal doses (MELA et al. 1977, PELTOMAA et al. 1979, TÄHTINEN 1979, SAARELA et al. 1981) and even if the dressed amounts have been weighed in spring (BAERUG 1977 b, HERNES 1978). When the potassium fertilizer has been applied in single dressings in spring the potassium content has been higher in the first cuts than in other cuts (RINNE et al. 1974). The drop in potassium content has been large on organogenic soils and on coarse mineral soils, but much less on clay soils (MELA et al. 1977, PELTOMAA et al. 1979).

Plant nitrogen contents (Table 4) varied between cuts in different stages of development in the same manner as the plant potassium contents. Differences between potassium rates were opposite to potassium content differences, as the available nitrogen was concentrated into the lessened amounts of plant tissue. A significant lowering of plant nitrogen content without any yield increase was observed in two cases.

Plant calcium and magnesium contents (Tables 5 and 6) usually decreased with increasing potassium rates, but to a very variable degree on different soils. In the first cuts the calcium and magnesium contents were not at all lowered by potassium fertilizer at sites where the soil clay content was $14 \%$ or more. The effect of potassium rates on plant calcium and magnesium contents increased with time, as the increase followed the potassium deficiency of the grass which became more and more severe.

The highest magnesium contents of plant observed in this study, up to 6.8 $\mathrm{g} / \mathrm{kg}$, are unusually high for a grass crop (RINNE et al. 1974, BAERUG $1977 \mathrm{~b}$, MELA et al. 1977, JOKINEN 1979, PELTOMAA et al. 1979, TÄHTINEN 1979, SAARELA et al. 1981). The changes in plant mineral composition have also 
Table 3. Potassium content of yield of timothy leys with increasing potessium fertilization rates $(\mathrm{g} / \mathrm{kg})$.

\begin{tabular}{|c|c|c|c|c|c|c|c|c|c|}
\hline \multirow[t]{2}{*}{$\mathrm{K}$ rate, $\mathrm{kg} / \mathrm{ha}$} & \multicolumn{9}{|c|}{ Trial } \\
\hline & 1 & 2 & 3 & 4 & 5 & 6 & 7 & 8 & 9 \\
\hline & \multicolumn{9}{|c|}{ 1st year 1 st cut } \\
\hline 0 & $21.4^{2}$ & $25.3^{2}$ & $13.8^{2}$ & $9.7^{2}$ & $15.3^{2}$ & $16.7^{a}$ & $32.1^{2}$ & 19.5 & $10.7^{a}$ \\
\hline 20 & $22.4^{\mathrm{ab}}$ & $27.9^{\mathrm{ab}}$ & $17.0^{\mathrm{ab}}$ & $11.2^{\mathrm{ab}}$ & $19.2^{\mathrm{b}}$ & $20.1^{2}$ & $34.8^{a}$ & 23.6 & $12.4^{2}$ \\
\hline 40 & $24.6^{\mathrm{ab}}$ & $27.9^{\mathrm{ab}}$ & $17.9^{b}$ & $13.2^{\mathrm{bc}}$ & $18.2^{\mathrm{ab}}$ & $21.3^{a}$ & $34.2^{a}$ & 28.9 & $14.9^{b}$ \\
\hline 60 & $25.5^{\mathrm{ab}}$ & $29.2^{\mathrm{b}}$ & $19.9^{\text {ac }}$ & $14.7^{\text {cd }}$ & $20.2^{\mathrm{bc}}$ & $27.8^{\mathrm{b}}$ & $38.1^{2}$ & $32.8^{a}$ & $16.7^{b}$ \\
\hline \multirow[t]{2}{*}{80} & $26.7^{b}$ & $30.5^{b}$ & $22.9^{c}$ & $17.3^{d}$ & $23.3^{c}$ & $29.2^{b}$ & $39.2^{\mathrm{a}}$ & $33.2^{2}$ & 19.2 \\
\hline & \multicolumn{9}{|c|}{1 st year 2 nd cut } \\
\hline 0 & 25.6 & $31.4^{2}$ & $12.6^{\mathrm{a}}$ & $6.7^{2}$ & 20.2 & $19.3^{a}$ & $28.0^{a}$ & $18.0^{a}$ & 11.8 \\
\hline 20 & $28.3^{2}$ & $30.1^{2}$ & $14.5^{\mathrm{ab}}$ & $9.0^{4}$ & 28.0 & $20.9^{\mathrm{ab}}$ & $30.3^{a b}$ & $19.6^{2}$ & 17.5 \\
\hline 40 & $29.5^{a}$ & $30.1^{a}$ & $19.1^{\mathrm{b}}$ & $13.4^{b}$ & 32.0 & $21.7^{\mathrm{ab}}$ & $29.4^{\mathrm{ab}}$ & $24.5^{b}$ & 23.9 \\
\hline 60 & $30.5^{a}$ & $30.8^{2}$ & $17.9^{b}$ & $16.2^{\mathrm{b}}$ & 35.3 & $26.5^{\mathrm{bc}}$ & $35.2^{\mathrm{b}}$ & $27.9^{b}$ & 29.9 \\
\hline \multirow[t]{2}{*}{80} & $31.4^{a}$ & $32.2^{2}$ & $25.1^{c}$ & 20.9 & 40.4 & $29.4^{c}$ & $36.9^{b}$ & $29.5^{b}$ & 35.8 \\
\hline & \multicolumn{9}{|c|}{2 nd year 1 st cut } \\
\hline 0 & $26.6^{2}$ & $29.9^{a}$ & $12.3^{\mathrm{a}}$ & 6.5 & $10.1^{2}$ & $15.8^{\mathrm{a}}$ & $23.5^{\mathrm{a}}$ & 17.7 & $8.8^{a}$ \\
\hline 20 & $27.6^{a}$ & $30.6^{\mathrm{a}}$ & $17.0^{\mathrm{ab}}$ & 11.0 & $12.8^{\mathrm{ab}}$ & $17.0^{\mathrm{ab}}$ & $25.0^{2}$ & 24.8 & $10.9^{a}$ \\
\hline 40 & $32.5^{b}$ & $32.3^{a b}$ & $21.1^{\mathrm{bc}}$ & 14.7 & $15.3^{\mathrm{b}}$ & $23.1^{\mathrm{b}}$ & $26.4^{a}$ & 32.2 & 15.3 \\
\hline 60 & $34.2^{b c}$ & $34.2^{\mathrm{bc}}$ & $23.9^{\text {cd }}$ & $21.3^{2}$ & 19.3 & $21.4^{\mathrm{ab}}$ & $21.7^{2}$ & 39.2 & $19.4^{\mathrm{b}}$ \\
\hline \multirow[t]{2}{*}{80} & $36.6^{c}$ & $36.3^{c}$ & $29.1^{d}$ & $22.8^{a}$ & 22.2 & $24.8^{\mathrm{b}}$ & 29.9 & 44.9 & $22.0^{b}$ \\
\hline & \multicolumn{9}{|c|}{ 2nd year 2nd cut } \\
\hline 0 & $22.4^{a}$ & $30.1^{\mathrm{a}}$ & 11.9 & 6.4 & 12.9 & $19.5^{2}$ & $31.8^{2}$ & 15.0 & 9.5 \\
\hline 20 & $27.1^{\mathrm{ab}}$ & $32.0^{a b}$ & $16.0^{\mathrm{a}}$ & 13.0 & 19.3 & $22.1^{\mathrm{ab}}$ & $37.0^{a b}$ & 19.7 & $20.6^{a}$ \\
\hline 40 & $28.7^{\mathrm{ab}}$ & $35.9^{\mathrm{bc}}$ & $19.1^{2}$ & $18.5^{\mathrm{a}}$ & 27.2 & $27.3^{\mathrm{ab}}$ & $38.6^{\mathrm{ab}}$ & 27.9 & $25.2^{2}$ \\
\hline 60 & $29.7^{\mathrm{ab}}$ & $36.5^{\mathrm{bc}}$ & 23.6 & $21.5^{a}$ & 31.9 & $28.1^{\mathrm{b}}$ & $39.5^{\mathrm{ab}}$ & 37.7 & 32.6 \\
\hline \multirow[t]{3}{*}{80} & $34.1^{\mathrm{b}}$ & $39.3^{c}$ & 29.9 & 29.3 & $40.4^{b}$ & $33.4^{b}$ & $43.3^{b}$ & 41.7 & 45.8 \\
\hline & \multicolumn{4}{|c|}{3 rd year trial 3} & \multicolumn{5}{|c|}{3 rd year trial 5} \\
\hline & 1 st cut & 2nd cut & & & 1 st cut & 2nd cut & & & \\
\hline 0 & 9.8 & $10.2^{2}$ & & & 6.0 & $6.9^{a}$ & & & \\
\hline 20 & $13.4^{2}$ & $13.9^{\mathrm{ab}}$ & & & 11.2 & $13.4^{\mathrm{ab}}$ & & & \\
\hline 40 & $15.8^{\mathrm{ab}}$ & $16.6^{2}$ & & & 15.9 & $19.2^{\mathrm{b}}$ & & & \\
\hline 60 & $18.6^{\mathrm{b}}$ & 21.1 & & & 20.1 & 28.0 & & & \\
\hline 80 & 23.3 & 30.0 & & & 25.8 & 41.4 & & & \\
\hline
\end{tabular}

varied between soil types in previous studies (MELA et al. 1977, TÄHTINEN 1979). On a heavy clay soil potassium fertilization has even increased the calcium and magnesium contents of timothy (SAARELA et al. 1981).

\section{Nutrient uptakes}

Potassium uptakes exceeded the amounts added in the fertilizer even with the highest rate (Table 7). Apparent recovery of applied potassium was nearly $100 \%$ on the non-clayey soils but lover on the clayey $(\geq 14 \%<0.002 \mathrm{~mm}$ ) soils in trials 1, 2 and 7 . The over $100 \%$ aparent recovery, which was significant in trial 9, is not impossible, as the more vigorous plants stimulated by applied potassium takes also soil potassium more efficiently.

Nitrogen uptakes also usually exceeded the nitrogen amouts $(160 \mathrm{~kg} \mathrm{~N} /$ 
Table 4. Nitrogen content of yield of timothy leys with increasing potassium fertilization rates $(\mathrm{g} / \mathrm{kg})$.

\begin{tabular}{|c|c|c|c|c|c|c|c|c|c|}
\hline \multirow[t]{2}{*}{$\mathrm{K}$ rate, $\mathrm{kg} / \mathrm{ha}$} & \multicolumn{9}{|c|}{ Trial } \\
\hline & 1 & 2 & 3 & 4 & 5 & 6 & 7 & 8 & 9 \\
\hline & \multicolumn{9}{|c|}{1 st year 1 st cut } \\
\hline 0 & $22.1^{2}$ & $21.8^{2}$ & $20.8^{2}$ & $24.0^{2}$ & $16.4^{2}$ & $28.5^{\mathrm{a}}$ & $36.7^{a}$ & $36.5^{2}$ & $24.9^{\mathrm{b}}$ \\
\hline 20 & $20.1^{2}$ & $21.2^{2}$ & $21.6^{2}$ & $22.5^{\mathrm{a}}$ & $17.2^{2}$ & $27.8^{2}$ & $34.2^{2}$ & $36.6^{2}$ & $22.5^{\mathrm{ab}}$ \\
\hline 40 & $21.7^{a}$ & $21.9^{2}$ & $21.9^{a}$ & $21.8^{a}$ & $15.9^{2}$ & $27.6^{2}$ & $32.0^{4}$ & $33.9^{2}$ & $21.6^{2}$ \\
\hline 60 & $22.3^{a}$ & $20.9^{2}$ & $22.9^{2}$ & $21.0^{a}$ & $16.4^{2}$ & $28.1^{2}$ & $34.9^{2}$ & $36.2^{2}$ & $20.7^{a}$ \\
\hline \multirow[t]{2}{*}{80} & $22.6^{2}$ & $22.1^{2}$ & $21.7^{2}$ & 20.1 & $16.3^{2}$ & $26.3^{2}$ & $35.9^{a}$ & $33.2^{2}$ & $20.8^{2}$ \\
\hline & \multicolumn{9}{|c|}{1 st year 2 nd cut } \\
\hline 0 & $25.7^{2}$ & $22.9^{2}$ & $22.6^{2}$ & 30.1 & $37.0^{2}$ & 23.6 & $27.0^{2}$ & $25.8^{\mathrm{b}}$ & 47.7 \\
\hline 20 & $24.7^{a}$ & $22.3^{4}$ & $21.7^{2}$ & $25.5^{\mathrm{a}}$ & $36.8^{2}$ & $21.9^{2}$ & $25.9^{a}$ & $23.9^{\mathrm{ab}}$ & 40.4 \\
\hline 40 & $24.6^{2}$ & $21.3^{2}$ & $22.6^{2}$ & $25.3^{2}$ & $38.0^{2}$ & $21.3^{2}$ & $26.3^{2}$ & $21.4^{2}$ & $36.9^{a}$ \\
\hline 60 & $25.5^{\mathrm{a}}$ & $23.1^{2}$ & $22.4^{2}$ & $22.0^{2}$ & $36.9^{2}$ & $21.5^{a}$ & $26.7^{2}$ & $22.2^{2}$ & $36.4^{2}$ \\
\hline \multirow[t]{2}{*}{80} & $23.9^{2}$ & $22.3^{2}$ & $21.7^{2}$ & $23.3^{2}$ & $37.8^{4}$ & $21.6^{2}$ & $25.9^{a}$ & $22.2^{2}$ & $34.5^{a}$ \\
\hline & \multicolumn{9}{|c|}{2 nd year 1 st cut } \\
\hline 0 & $27.5^{a}$ & $25.4^{2}$ & $29.0^{c}$ & $34.4^{b}$ & $16.9^{\circ}$ & $25.1^{2}$ & $28.2^{2}$ & $32.8^{\mathrm{b}}$ & 26.3 \\
\hline 20 & $27.6^{2}$ & $26.1^{2}$ & $27.8^{\mathrm{bc}}$ & $28.1^{\mathrm{ab}}$ & $15.2^{\mathrm{bc}}$ & $25.5^{\mathrm{a}}$ & $27.8^{\mathrm{a}}$ & $30.8^{\mathrm{ab}}$ & 21.4 \\
\hline 40 & $27.1^{2}$ & $26.6^{2}$ & $25.7^{2}$ & $27.4^{\text {ab }}$ & $14.4^{b}$ & $24.0^{2}$ & $27.2^{2}$ & $27.9^{2}$ & $18.4^{2}$ \\
\hline 60 & $26.0^{2}$ & $24.6^{\mathrm{a}}$ & $25.0^{2}$ & $24.5^{2}$ & $13.9^{\mathrm{ab}}$ & $23.5^{\mathrm{a}}$ & $27.6^{\mathrm{a}}$ & $29.4^{2}$ & $18.9^{\prime}$ \\
\hline \multirow[t]{2}{*}{80} & $26.8^{2}$ & $24.3^{2}$ & $26.2^{\text {ab }}$ & $24.9^{a}$ & $12.8^{a}$ & $25.9^{a}$ & 25.2 & $28.3^{2}$ & 16.6 \\
\hline & \multicolumn{9}{|c|}{ 2nd year 2 nd cut } \\
\hline 0 & $29.8^{2}$ & $28.8^{b}$ & 30.1 & 36.9 & $39.7^{b}$ & $28.0^{4}$ & $29.6^{\mathrm{a}}$ & 30.2 & 41.6 \\
\hline 20 & $26.8^{2}$ & $27.7^{\mathrm{ab}}$ & $25.5^{\mathrm{a}}$ & 27.2 & $35.9^{\mathrm{b}}$ & $25.7^{2}$ & $29.3^{2}$ & 23.7 & $35.5^{\mathrm{b}}$ \\
\hline 40 & $27.0^{2}$ & $27.5^{\mathrm{ab}}$ & $26.2^{2}$ & $22.7^{2}$ & $30.6^{2}$ & $25.1^{2}$ & $29.1^{2}$ & $21.1^{2}$ & $32.7^{\mathrm{ab}}$ \\
\hline 60 & $27.9^{a}$ & $26.5^{a}$ & $24.9^{2}$ & $21.5^{2}$ & $27.0^{2}$ & $23.9^{2}$ & $28.6^{a}$ & $20.2^{2}$ & $30.6^{2}$ \\
\hline \multirow[t]{3}{*}{80} & $26.7^{2}$ & $26.8^{a}$ & $24.3^{\mathrm{a}}$ & $21.6^{2}$ & $28.1^{2}$ & $24.1^{2}$ & $29.0^{a}$ & $20.2^{2}$ & $30.0^{a}$ \\
\hline & \multicolumn{4}{|c|}{3 rd year trial 3} & \multicolumn{5}{|c|}{ 3rd year trial 5} \\
\hline & 1 st cut & 2nd cut & & & 1 st cut & 2nd cut & & & \\
\hline 0 & $25.0^{\mathrm{b}}$ & 32.5 & & & 29.4 & $35.2^{c}$ & & & \\
\hline 20 & $23.3^{\mathrm{ab}}$ & 28.2 & & & 22.6 & $31.7^{\mathrm{bc}}$ & & & \\
\hline 40 & $19.9^{4}$ & $23.8^{2}$ & & & $18.6^{\mathrm{b}}$ & $27.5^{\mathrm{ab}}$ & & & \\
\hline 60 & $20.0^{2}$ & $23.4^{2}$ & & & $17.2^{\mathrm{ab}}$ & $24.9^{a}$ & & & \\
\hline 80 & $20.8^{2}$ & $22.8^{2}$ & & & $15.9^{a}$ & $23.9^{a}$ & & & \\
\hline
\end{tabular}

ha/year) added in the fertilizer (Table 7). This was a result of the high content of organic matter in the soils and nitrogen mobilization from it. Only severe potassium deficiency decreased nitrogen uptake noticeably, as the increase in yield nitrogen content compensated the decrease in dry matter yield with slight deficiency.

Calcium and magnesium uptakes were not much affected by potassium rates except in the case of very severe deficiencies, when the increases in contentswere not large enough to compensate the decreases in yields (Table 7). Calcium and magnesium uptakes were maximum with slight deficient potassium rates, which were the middle rates on most of the soils.

Potassium uptakes without potassium application were small and soon decreased on non-clayey soils, but were larger on clayey soils with a larger content of acid-extractable potassium (Table 8). 
Table 5. Calcium content of yield of timothy leys with increasing potassium fertilization rates $(\mathrm{g} / \mathrm{kg})$.

\begin{tabular}{|c|c|c|c|c|c|c|c|c|c|}
\hline \multirow[t]{2}{*}{$\mathrm{K}$ rate, $\mathrm{kg} / \mathrm{ha}$} & \multicolumn{9}{|c|}{ Trial } \\
\hline & 1 & 2 & 3 & 4 & 5 & 6 & 7 & 8 & 9 \\
\hline & \multicolumn{9}{|c|}{1 st year 1 st cut } \\
\hline 0 & $2.3^{a}$ & $2.5^{2}$ & $3.8^{2}$ & $2.7^{2}$ & $2.7^{a}$ & $7.1^{b}$ & $3.3^{2}$ & $4.3^{b}$ & $5.8^{\mathrm{b}}$ \\
\hline 20 & $2.7^{\mathrm{a}}$ & $2.4^{2}$ & $3.2^{2}$ & $2.6^{2}$ & $2.5^{\mathrm{a}}$ & $5.9^{2}$ & $3.3^{2}$ & $3.5^{\mathrm{ab}}$ & $5.3^{\mathrm{ab}}$ \\
\hline 40 & $2.6^{2}$ & $2.4^{2}$ & $4.0^{2}$ & $4.0^{2}$ & $2.4^{a}$ & $6.0^{\mathrm{ab}}$ & $2.7^{2}$ & $3.4^{\mathrm{ab}}$ & $5.1^{\mathrm{ab}}$ \\
\hline 60 & $2.8^{a}$ & $2.4^{2}$ & $3.7^{\mathrm{a}}$ & $2.3^{\mathrm{a}}$ & $2.5^{\mathrm{a}}$ & $6.0^{a b}$ & $3.0^{a}$ & $3.0^{4}$ & $3.8^{a}$ \\
\hline \multirow[t]{2}{*}{80} & $2.6^{\mathrm{a}}$ & $2.6^{2}$ & $3.2^{2}$ & $2.1^{2}$ & $2.6^{4}$ & $4.9^{4}$ & $3.0^{2}$ & $2.9^{a}$ & $4.4^{\mathrm{ab}}$ \\
\hline & \multicolumn{9}{|c|}{1 st year 2 nd cut } \\
\hline 0 & $3.0^{a}$ & $2.5^{\mathrm{a}}$ & $4.6^{\mathrm{a}}$ & $4.7^{u}$ & $5.1^{2}$ & $8.1^{2}$ & $3.1^{2}$ & $3.9^{\mathrm{b}}$ & 9.2 \\
\hline 20 & $2.8^{\mathrm{a}}$ & $2.5^{\mathrm{a}}$ & $4.6^{2}$ & $4.2^{\mathrm{bc}}$ & $4.4^{a}$ & $7.8^{\mathrm{a}}$ & $2.9^{2}$ & $3.7^{\mathrm{a}}$ & $8.1^{2}$ \\
\hline 40 & $2.6^{2}$ & $2.3^{\mathrm{a}}$ & $4.9^{4}$ & $3.8^{\mathrm{ab}}$ & $4.4^{2}$ & $8.1^{2}$ & $3.1^{2}$ & $3.5^{\mathrm{a}}$ & $7.6^{\mathrm{a}}$ \\
\hline 60 & $2.9^{\mathrm{a}}$ & $3.1^{2}$ & $4.4^{2}$ & $3.2^{\mathrm{a}}$ & $4.5^{\mathrm{a}}$ & $7.6^{2}$ & $3.1^{2}$ & $3.5^{\mathrm{a}}$ & 7.0 \\
\hline \multirow[t]{2}{*}{80} & $2.8^{\mathrm{a}}$ & $2.8^{\mathrm{a}}$ & $4.3^{a}$ & $3.2^{2}$ & $4.3^{\mathrm{a}}$ & $7.2^{2}$ & $2.9^{a}$ & $3.3^{2}$ & 5.9 \\
\hline & \multicolumn{9}{|c|}{2 nd year 1 st cut } \\
\hline 0 & $3.6^{\mathrm{b}}$ & $3.2^{\mathrm{ab}}$ & $6.9^{b}$ & $6.6^{c}$ & $3.8^{\mathrm{b}}$ & $4.0^{2}$ & $2.3^{2}$ & $5.7^{c}$ & 7.3 \\
\hline 20 & $3.7^{b}$ & $3.7^{b}$ & $6.1^{\mathrm{bb}}$ & $5.8^{\mathrm{bc}}$ & $3.6^{\mathrm{ab}}$ & $4.9^{2}$ & $2.2^{2}$ & $5.0^{\mathrm{bc}}$ & 6.0 \\
\hline 40 & $3.4^{\mathrm{ab}}$ & $3.2^{2 b}$ & $4.9^{2}$ & $5.1^{\mathrm{ab}}$ & $3.3^{\mathrm{b}}$ & $4.4^{2}$ & $1.7^{\mathrm{a}}$ & $4.6^{\mathrm{ab}}$ & $5.3^{2}$ \\
\hline 60 & $3.6^{2}$ & $3.2^{\mathrm{ab}}$ & $5.2^{2}$ & $4.3^{a}$ & $3.3^{2}$ & $4.8^{2}$ & $2.0^{2}$ & $4.2^{2}$ & $5.1^{2}$ \\
\hline \multirow[t]{2}{*}{80} & $3.0^{a}$ & $2.7^{a}$ & $4.8^{a}$ & $4.4^{2}$ & 2.8 & $3.9^{a}$ & $2.1^{2}$ & $4.1^{2}$ & 4.3 \\
\hline & \multicolumn{9}{|c|}{ 2nd year 2nd cut } \\
\hline 0 & $5.1^{2}$ & $4.0^{2}$ & $5.3^{2}$ & 7.0 & 6.9 & $6.6^{2}$ & $4.9^{2}$ & 5.3 & $10.0^{2}$ \\
\hline 20 & $4.5^{\mathrm{a}}$ & $3.7^{2}$ & $4.7^{2}$ & 6.2 & $6.2^{c}$ & $7.1^{2}$ & $4.6^{\mathrm{a}}$ & 4.3 & $9.9^{\mathrm{a}}$ \\
\hline 40 & $4.3^{a}$ & $3.6^{2}$ & $4.6^{2}$ & $5.1^{2}$ & $5.7^{\mathrm{bc}}$ & $6.4^{2}$ & $4.5^{\mathrm{a}}$ & $3.8^{\mathrm{a}}$ & 9.3 \\
\hline 60 & $4.6^{\mathrm{a}}$ & $3.5^{\mathrm{a}}$ & $4.1^{2}$ & $4.7^{a}$ & $5.1^{\mathrm{ab}}$ & $6.3^{2}$ & $3.9^{a}$ & $3.3^{2}$ & $8.4^{2}$ \\
\hline \multirow[t]{3}{*}{80} & $4.0^{2}$ & $3.5^{\mathrm{a}}$ & $4.7^{a}$ & $4.9^{a}$ & $4.6^{2}$ & $4.9^{2}$ & $4.1^{2}$ & $3.3^{\mathrm{a}}$ & $7.9^{2}$ \\
\hline & \multicolumn{4}{|c|}{3 rd year trial 3} & \multicolumn{5}{|c|}{ 3rd year trial 5} \\
\hline & 1st cut & 2nd cut & & & 1st cut & 2nd cut & & & \\
\hline 0 & $3.6^{b}$ & $4.9^{2}$ & & & 6.4 & 10.0 & & & \\
\hline 20 & $3.1^{\mathrm{ab}}$ & $5.5^{a}$ & & & $5.2^{\mathrm{b}}$ & $7.6^{2}$ & & & \\
\hline 40 & $2.7^{2}$ & $5.5^{2}$ & & & $4.6^{\mathrm{ab}}$ & $6.8^{\mathrm{a}}$ & & & \\
\hline 60 & $2.8^{a}$ & $4.0^{a}$ & & & $4.1^{2}$ & $8.0^{2}$ & & & \\
\hline 80 & $2.4^{a}$ & $4.4^{2}$ & & & $3.2^{2}$ & $7.0^{2}$ & & & \\
\hline
\end{tabular}

Soil potassium

At the end of the trials the soils were quite exhausted of available potassium even with the highest rates (Table 9). This would be expected after the negative balances. The subsurface soils at a depth of 20 to $40 \mathrm{~cm}$ were relatively more depleted than the surface soils and the potassium fertilizer had no effect on them. In the surface soils the contents of potassium extractable in acid ammonium acetate were highest with the largest rate, but the differences were usually small, in accordance to the high apparent recoveries in yield. The soil effects were largest in trials 1 and 2 and 7, where the differences in uptakes were smallest. In trial 4 the soil samples were taken after the third year, when oat was grown with a positive potassium balance up to $40 \mathrm{~kg} \mathrm{~K} / \mathrm{ha}$ with the highest rate (results not given here). 
Table 6. Magnesium content of yield of timothy leys with increasing potassium fertilization rates $(\mathrm{g} / \mathrm{kg})$.

\begin{tabular}{|c|c|c|c|c|c|c|c|c|c|}
\hline \multirow[t]{2}{*}{$\mathrm{K}$ rate, $\mathrm{kg} / \mathrm{ha}$} & \multicolumn{9}{|c|}{ Trial } \\
\hline & 1 & 2 & 3 & 4 & 5 & 6 & 7 & 8 & 9 \\
\hline & \multicolumn{9}{|c|}{ 1st year 1 st cut } \\
\hline 0 & $0.8^{2}$ & $0.8^{a}$ & $1.2^{\mathrm{a}}$ & $2.4^{4}$ & $1.5^{c}$ & 2.8 & $2.1^{2}$ & 2.9 & $2.2^{c}$ \\
\hline 20 & $0.8^{2}$ & $0.7^{2}$ & $1.0^{a}$ & $2.3^{\mathrm{c}}$ & $1.5^{4}$ & $2.4^{2}$ & $2.0^{4}$ & $2.4^{2}$ & $1.8^{\mathrm{bc}}$ \\
\hline 40 & $0,8^{a}$ & $0.7^{2}$ & $1.1^{2}$ & $2.1^{b c}$ & $1.5^{2}$ & $2.2^{2}$ & $2.0^{a}$ & $2.0^{2}$ & $1.6^{\text {ab }}$ \\
\hline 60 & $0.8^{2}$ & $0.7^{2}$ & $1.0^{2}$ & $1.8^{\mathrm{ab}}$ & $1.3^{2}$ & $2.2^{2}$ & $1.9^{2}$ & $1.9^{2}$ & $1,1^{2}$ \\
\hline \multirow[t]{2}{*}{80} & $0.8^{2}$ & $0.8^{2}$ & $0.8^{2}$ & $1.6^{4}$ & $1.3^{2}$ & 1.8 & $1.7^{2}$ & $1.8^{2}$ & $1.2^{2}$ \\
\hline & \multicolumn{9}{|c|}{1 st year 2 nd cut } \\
\hline 0 & $1.0^{2}$ & $0.9^{2}$ & $1.5^{\mathrm{b}}$ & 3.2 & 3.8 & $3.2^{\mathrm{b}}$ & $2.0^{4}$ & $2.9^{b}$ & 3.5 \\
\hline 20 & $1.0^{a}$ & $0.8^{\mathrm{a}}$ & $1.4^{\mathrm{ab}}$ & 2.8 & $3.1^{2}$ & $2.9^{a b}$ & $1.9^{2}$ & $2.6^{\mathrm{b}}$ & 2.9 \\
\hline 40 & $0.9^{a}$ & $0.8^{2}$ & $1.4^{\mathrm{ab}}$ & 2.3 & $3.1^{\mathrm{a}}$ & $2.8^{\mathrm{ab}}$ & $1.9^{a}$ & $2.1^{2}$ & 2.5 \\
\hline 60 & $0.9^{2}$ & $0.9^{2}$ & $1.2^{\mathrm{ab}}$ & $1.8^{2}$ & $2.9^{2}$ & $2.6^{\mathrm{ab}}$ & $1.9^{2}$ & $2.0^{a}$ & 2.1 \\
\hline \multirow[t]{2}{*}{80} & $0.9^{2}$ & $0.9^{a}$ & $1.1^{2}$ & $1.7^{2}$ & $2.5^{\mathrm{a}}$ & $2.3^{a}$ & $1.7^{2}$ & $1.8^{2}$ & 1.8 \\
\hline & \multicolumn{9}{|c|}{ 2nd year 1 st cut } \\
\hline 0 & $1.1^{\mathrm{c}}$ & $1.0^{\mathrm{b}}$ & 2.4 & 4.9 & 2.4 & $2.0^{2}$ & $1.3^{2}$ & 4.2 & 2.9 \\
\hline 20 & $1.0^{\mathrm{bc}}$ & $1.0^{b}$ & 2.0 & 3.6 & $2.0^{\mathrm{b}}$ & $2.4^{2}$ & $1.2^{2}$ & 3.5 & 2.2 \\
\hline 40 & $1.0^{\mathrm{bc}}$ & $0.9^{\mathrm{ab}}$ & $1.5^{\mathrm{a}}$ & 3.2 & $1.8^{b}$ & $1.9^{2}$ & $0.9^{2}$ & $2.8^{\mathrm{b}}$ & 1.8 \\
\hline 60 & $0.9^{\mathrm{ab}}$ & $0.9^{\mathrm{ab}}$ & $1.5^{4}$ & $2.3^{2}$ & $1.6^{\mathrm{ab}}$ & $2.0^{2}$ & $1.1^{2}$ & $2.6^{\mathrm{ab}}$ & 1.5 \\
\hline \multirow[t]{2}{*}{80} & $0.8^{2}$ & $0.8^{a}$ & $1.3^{2}$ & $2.2^{2}$ & $1.3^{\mathrm{a}}$ & 1.2 & $1.0^{2}$ & $2.3^{2}$ & 1.2 \\
\hline & \multicolumn{9}{|c|}{2 nd year 2 nd cut } \\
\hline 0 & $1.4^{\mathrm{b}}$ & $1.4^{2}$ & 2.3 & 5.1 & 4.7 & $2.8^{\mathrm{b}}$ & $3.2^{2}$ & 5.0 & 5.8 \\
\hline 20 & $1.2^{\mathrm{ab}}$ & $1.2^{2}$ & $1.8^{2}$ & 3.9 & $3.9^{c}$ & $3.1^{\mathrm{b}}$ & $3.0^{\circ}$ & 3.7 & 4.7 \\
\hline 40 & $1.1^{\mathrm{ab}}$ & $1.1^{2}$ & $1.8^{2}$ & $3.0^{b}$ & $3.2^{2 \mathrm{bb}}$ & $2.6^{\mathrm{b}}$ & $2.6^{2}$ & 3.1 & 4.1 \\
\hline 60 & $1.2^{\mathrm{ab}}$ & $1.1^{2}$ & $1.4^{\mathrm{a}}$ & $2.4^{\mathrm{ab}}$ & $2.7^{\mathrm{ab}}$ & $2.3^{\mathrm{ab}}$ & $2.3^{2}$ & $2.3^{2}$ & $3.3^{2}$ \\
\hline \multirow[t]{3}{*}{80} & $1.0^{2}$ & $1.1^{2}$ & $1.4^{2}$ & $2.1^{2}$ & $2.1^{2}$ & $1.7^{2}$ & $2.4^{2}$ & $2.2^{2}$ & $2.8^{2}$ \\
\hline & \multicolumn{4}{|c|}{3 rd year trial 3} & \multicolumn{5}{|c|}{3 rd year trial 5} \\
\hline & 1 st cut & 2 nd cut & & & 1 st cut & 2 nd cut & & & \\
\hline 0 & $1.5^{\mathrm{b}}$ & $2.7^{\circ}$ & & & 4.3 & 6.8 & & & \\
\hline 20 & $1.3^{b}$ & $2.2^{\mathrm{be}}$ & & & $3.1^{\mathrm{b}}$ & $4.7^{2}$ & & & \\
\hline 40 & $1.0^{2}$ & $2.3^{b c}$ & & & $2.5^{\mathrm{ab}}$ & $3.7^{2}$ & & & \\
\hline 60 & $1.0^{2}$ & $1.7^{\mathrm{ab}}$ & & & $2.1^{2}$ & $4.0^{2}$ & & & \\
\hline 80 & $0.8^{a}$ & $1.3^{\mathrm{a}}$ & & & 1.5 & $3.1^{2}$ & & & \\
\hline
\end{tabular}

The exhaustion of available potassium reserves of soil is most rapid with heavy nitrogen dressings on peat soils (SILLANPÄÄ and RINNE 1975). The potassium deletion of the subsurface layers (Table 9) show that timothy takes up potassium from below the plough layer efficiently, at least under some conditions. The proportion of potassium taken up below the plough layer may be greater than 5-10\%, which is an estimation by JOY et al. (1973).

Dependence of response on soil and plant variables

The relative differences between the dry matter yields were greater when there were less extractable potassium (K-AAA) in soil, less potassium in plant and more magnesium in plant (Table 10). K-HCL was not accepted to 
Table 7. Nutrient uptakes of timothy with increasing potassium rates $(\mathrm{kg} / \mathrm{ha} / \mathrm{year}$ except recovery). Means of the first two years.

$\mathrm{K}$ rate, $\mathrm{kg} / \mathrm{ha}$ Trial

\begin{tabular}{lllllllll}
\hline 1 & 2 & 3 & 4 & 5 & 6 & 7 & 8 & 9
\end{tabular}

\begin{tabular}{|c|c|c|c|c|c|c|c|c|c|}
\hline \multirow[b]{2}{*}{0} & \multicolumn{9}{|c|}{ Potassium } \\
\hline & $297^{2}$ & $347^{2}$ & 89 & 42 & 85 & $121^{2}$ & $231^{2}$ & 76 & 34 \\
\hline 40 & $333^{\text {ab }}$ & $370^{a b}$ & 141 & 76 & 126 & $144^{\mathrm{ab}}$ & $263^{\mathrm{ab}}$ & 117 & 73 \\
\hline 80 & $356^{b}$ & $390^{b}$ & $184^{a}$ & 120 & 161 & $177^{\mathrm{bc}}$ & $267^{\mathrm{ab}}$ & 172 & 124 \\
\hline 120 & $371^{b c}$ & $391^{\mathrm{b}}$ & $210^{a}$ & 159 & 192 & $211^{\mathrm{cd}}$ & $289^{\mathrm{ab}}$ & 209 & 168 \\
\hline 160 & $416^{c}$ & $402^{b}$ & 260 & 190 & 231 & $234^{d}$ & $307^{d}$ & 233 & 227 \\
\hline
\end{tabular}

Effects of potassium fertilization on potassium uptake (Uptake-uptake with $\mathrm{K}$ rate 0 )

\begin{tabular}{|c|c|c|c|c|c|c|c|c|c|}
\hline 0 & 0 & 0 & 0 & 0 & 0 & 0 & 0 & 0 & 0 \\
\hline 40 & 36 & 23 & 52 & 34 & 41 & 23 & 32 & 41 & 39 \\
\hline 80 & 59 & 43 & 95 & 78 & 76 & 56 & 36 & 96 & 90 \\
\hline 120 & 74 & 44 & 121 & 117 & 107 & 90 & 58 & 133 & 134 \\
\hline 160 & 119 & 55 & 171 & 148 & 146 & 122 & 76 & 157 & 193 \\
\hline & \multicolumn{9}{|c|}{$\%$ recovered of fertilizer potassium with confidence limits $(0.05)$} \\
\hline 40 & $90 \pm 45$ & $58 \pm 48$ & $130 \pm 45$ & $85 \pm 30$ & $103 \pm 50$ & $58 \pm 58$ & $80 \pm 75$ & $103 \pm 38$ & $98 \pm 28$ \\
\hline 80 & $75 \pm 23$ & $54 \pm 24$ & $119 \pm 23$ & $98 \pm 15$ & $95 \pm 25$ & $70 \pm 29$ & $45 \pm 29$ & $120 \pm 19$ & $113 \pm 14$ \\
\hline 120 & $62 \pm 15$ & $37 \pm 16$ & $101 \pm 15$ & $98 \pm 10$ & $89 \pm 17$ & $75 \pm 19$ & $48 \pm 25$ & $111 \pm 13$ & $112 \pm 9$ \\
\hline 160 & $70 \pm 11$ & $34 \pm 12$ & $107 \pm 11$ & $93 \pm 8$ & $90 \pm 13$ & $76 \pm 14$ & $48 \pm 19$ & $98 \pm 10$ & $121 \pm 7$ \\
\hline & \multicolumn{9}{|c|}{ Nitrogen } \\
\hline 0 & $324^{a}$ & $294^{b}$ & 174 & $156^{2}$ & 136 & $176^{2}$ & $239^{2}$ & 124 & 97 \\
\hline 40 & $319^{2}$ & $299^{\mathrm{b}}$ & 212 & $174^{\mathrm{ab}}$ & $157^{2}$ & $180^{4}$ & $246^{2}$ & $149^{a}$ & 141 \\
\hline 80 & $315^{2}$ & $302^{2}$ & $232^{2}$ & $193^{c}$ & $163^{a}$ & $187^{2}$ & $236^{2}$ & $152^{2}$ & $169^{a}$ \\
\hline 120 & $320^{4}$ & $286^{\mathrm{ab}}$ & $239^{2}$ & $193^{c}$ & $159^{2}$ & $194^{2}$ & $239^{4}$ & $156^{\mathrm{a}}$ & $181^{\mathrm{ab}}$ \\
\hline 160 & $328^{2}$ & $275^{2}$ & $226^{a}$ & $191^{c}$ & $161^{2}$ & $200^{4}$ & $234^{2}$ & $155^{a}$ & $189^{b}$ \\
\hline & \multicolumn{9}{|c|}{ Calcium } \\
\hline 0 & $43^{2}$ & $37^{2}$ & 35 & 25 & $24^{2}$ & $44^{2}$ & $27^{2}$ & 19 & 23 \\
\hline 40 & $44^{a}$ & $38^{a}$ & $42^{2}$ & $31^{2}$ & $28^{2}$ & $47^{a}$ & $28^{a}$ & $22^{a}$ & 35 \\
\hline 80 & $40^{2}$ & $36^{2}$ & $45^{2}$ & $32^{2}$ & $29^{4}$ & $49^{a}$ & $27^{2}$ & $24^{2}$ & $43^{4}$ \\
\hline 120 & $43^{2}$ & $37^{2}$ & $43^{2}$ & $31^{2}$ & $29^{2}$ & $49^{2}$ & $25^{2}$ & $22^{2}$ & $41^{2}$ \\
\hline 160 & $40^{4}$ & $35^{a}$ & $42^{2}$ & $30^{a}$ & $27^{a}$ & $43^{4}$ & $25^{a}$ & $22^{a}$ & $43^{2}$ \\
\hline & \multicolumn{9}{|c|}{ Magnesium } \\
\hline 0 & $14^{a}$ & $12^{\mathrm{b}}$ & $12^{\mathrm{A}}$ & $19^{a b}$ & $16^{\mathrm{b}}$ & $18^{2}$ & $17^{a}$ & $14^{\mathrm{ab}}$ & 9 \\
\hline 40 & $13^{a}$ & $12^{b}$ & $14^{b c}$ & $21^{b}$ & $17^{b}$ & $19^{a}$ & $17^{a}$ & $17^{c}$ & $13^{a}$ \\
\hline 80 & $12^{2}$ & $11^{\mathrm{ab}}$ & $15^{c}$ & $21^{\mathrm{b}}$ & $17^{b}$ & $18^{2}$ & $16^{2}$ & $16^{\mathrm{bc}}$ & $15^{b}$ \\
\hline 120 & $12^{\mathrm{a}}$ & $11^{\mathrm{ab}}$ & $13^{\mathrm{ab}}$ & $18^{\mathrm{ab}}$ & $15^{\mathrm{ab}}$ & $18^{a}$ & $15^{\mathrm{a}}$ & $14^{\mathrm{ab}}$ & $13^{\mathrm{ab}}$ \\
\hline 160 & $11^{2}$ & $10^{a}$ & $12^{a}$ & $16^{a}$ & $13^{2}$ & $15^{2}$ & $14^{2}$ & $13^{2}$ & $13^{2}$ \\
\hline
\end{tabular}

regression calculus because of too abnormal a distribution of the values. Plant $\mathrm{Ca}$ is related to $\mathrm{K}$ deficiency in the same manner as plant $\mathrm{Mg}$ and could substitute for it, but does not give much additional information. The influence of $\mathrm{Mg}$ to $\mathrm{K}$ nutrition may not usually be as important as it seems to be in the present material with very severe potassium deficiencies.

When the $N / K$ ratio of plant was substituted by 1 /plant $K$ and plant $N$, the $\mathrm{R}$ square values were not much changed. The ratio is, however, when reserved to $\mathrm{K} / \mathrm{N}$, easier to apply in practice. The relative yields of the rates 
Table 8 . Potassium uptake by timothy yields without $\mathrm{K}$ fertilization $(\mathrm{kg} / \mathrm{ha} / \mathrm{year})$.

\begin{tabular}{lccrrrrr}
\hline \multirow{2}{*}{ Trial } & \multirow{2}{*}{ Org. C $\%$} & Clay $(<0.002 \mathrm{~mm})$ & \multicolumn{2}{c}{ Soil test values } & \multicolumn{3}{c}{ Potassium uptake } \\
& & & $\mathrm{K}_{\mathrm{AAA}}$ & $\mathrm{K}_{\mathrm{HCl}}$ & 1st $\mathrm{yr}$ & 2nd $\mathrm{yr}$ & 3rd $\mathrm{yr}$ \\
\hline 1 & 7.0 & 14 & 102 & 1648 & 278 & 315 & \\
2 & 7.5 & 16 & 149 & 1910 & 295 & 399 & \\
3 & 39 & - & 73 & 148 & 109 & 68 & 50 \\
4 & 38 & - & 34 & 74 & 58 & 25 & \\
5 & 4.2 & 3 & 86 & 158 & 102 & 69 & 17 \\
6 & 13 & - & 46 & 199 & 141 & 101 & \\
7 & 3.5 & 33 & 102 & 1960 & 265 & 197 & \\
8 & 24 & - & 49 & 95 & 110 & 41 & \\
9 & 35 & - & 44 & 70 & 52 & 17 & \\
\hline
\end{tabular}

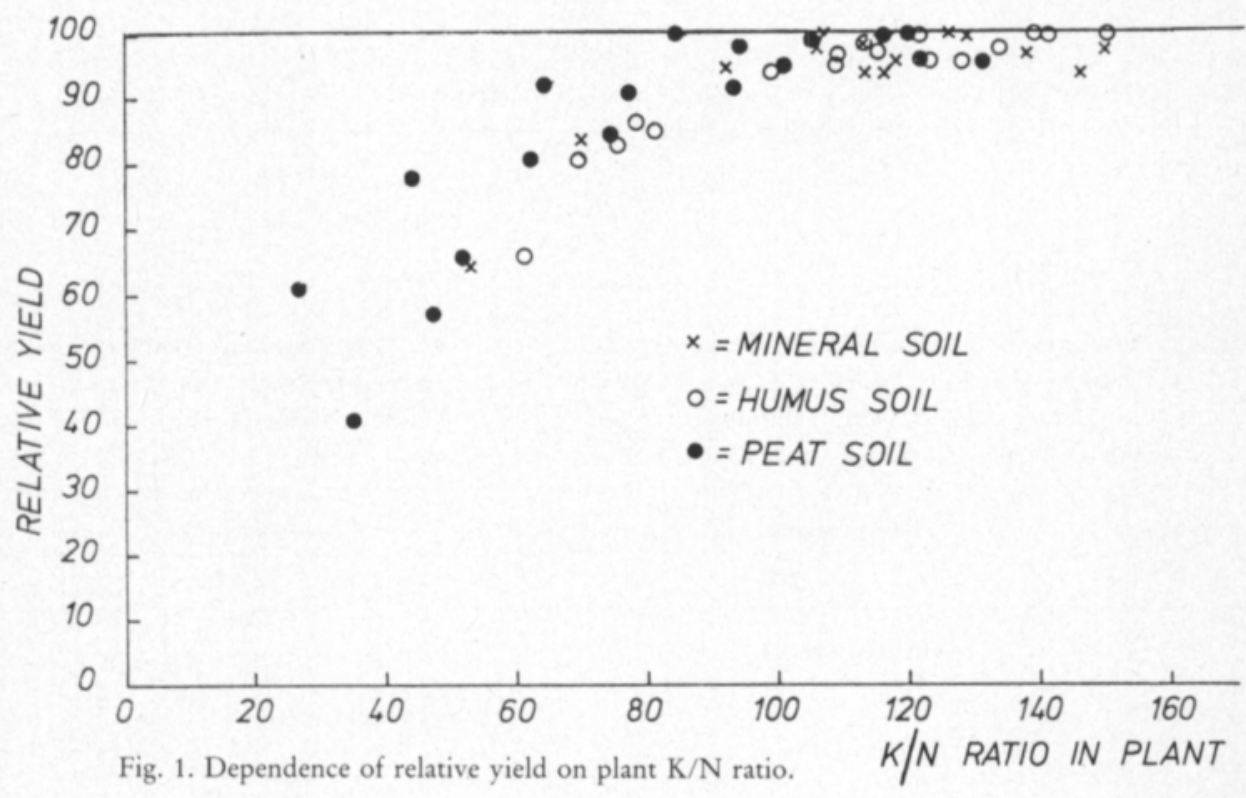

(highest $=100$ ) are plotted against the mean $\mathrm{K} / \mathrm{N}$ ratios in Figure 1. The figure shows that yield is likely to begin decreasing when the $\mathrm{K} / \mathrm{N}$ ratio decreases under 1 . The most deviating plots above the others are from trial 4 , where the herbage contained the most wild grass species.

\section{Discussion}

The results confirm the importance of potassium fertilization. for ley crops on Finnish peat soils. In the 42 long-term field trials in the years 1932 to 1959 on peat soils, $120 \mathrm{~kg} / \mathrm{ha}$ potassium was required for full yields although the nitrogen fertilization was $30 \mathrm{~kg} / \mathrm{ha}$ only and the level of yields 
Table 9. "Exchangeable" soil potassium (K-AAA) after 2-3 years potassium fertilization treatments (the same test values ao the beginning of trials given in brackets).

\begin{tabular}{|c|c|c|c|c|c|c|c|c|c|}
\hline \multirow[t]{2}{*}{$\mathrm{K}$ rate, $\mathrm{kg} / \mathrm{ha} / \mathrm{yr}$} & \multicolumn{9}{|c|}{ Trial } \\
\hline & 1 & 2 & 3 & 4 & 5 & 6 & 7 & 8 & 9 \\
\hline & \multicolumn{9}{|c|}{ Surface soil $(0-20 \mathrm{~cm})$} \\
\hline & $(102)$ & (149) & (73) & (34) & $(86)$ & $(46)$ & (102) & (49) & (44) \\
\hline 0 & $53^{a}$ & $59^{a}$ & $35^{2}$ & $25^{a}$ & $11^{2}$ & $31^{2}$ & $65^{a}$ & $21^{2}$ & $29^{2}$ \\
\hline 40 & $60^{\mathrm{ab}}$ & $77^{\mathrm{ab}}$ & $35^{2}$ & $25^{2}$ & $15^{\mathrm{ab}}$ & $33^{a}$ & $73^{2}$ & $23^{2}$ & $25^{a}$ \\
\hline 80 & $65^{\mathrm{ab}}$ & $63^{a}$ & $43^{a}$ & $30^{2}$ & $14^{a}$ & $33^{a}$ & $65^{a}$ & $26^{2}$ & $23^{2}$ \\
\hline 120 & $75^{b}$ & $80^{\mathrm{ab}}$ & $45^{\mathrm{ab}}$ & $30^{2}$ & $19^{\mathrm{b}}$ & $35^{4}$ & $73^{a}$ & $23^{2}$ & $29^{2}$ \\
\hline \multirow[t]{3}{*}{160} & $73^{b}$ & $93^{b}$ & $55^{b}$ & 53 & $21^{\mathrm{b}}$ & 47 & 103 & 33 & $30^{4}$ \\
\hline & \multicolumn{9}{|c|}{ Subsurface soil $(20-40 \mathrm{~cm})$} \\
\hline & (86) & $(92)$ & $(59)$ & (28) & (18) & (15) & (49) & (22) & (23) \\
\hline 0 & $60^{a}$ & $75^{a}$ & $-a^{2}$ & $15^{a}$ & $10^{2}$ & $19^{a}$ & $40^{2}$ & $13^{a}$ & $12^{2}$ \\
\hline 40 & $68^{a}$ & $83^{a}$ & - & $16^{2}$ & $10^{2}$ & $16^{a}$ & $33^{a}$ & $10^{a}$ & $10^{2}$ \\
\hline 80 & $65^{a}$ & $80^{4}$ & - & $19^{2}$ & $9^{2}$ & $23^{a}$ & $43^{a}$ & $13^{a}$ & $10^{2}$ \\
\hline 120 & $66^{2}$ & $73^{a}$ & - & $14^{a}$ & $9^{a}$ & $15^{a}$ & $30^{a}$ & $11^{2}$ & $10^{a}$ \\
\hline 160 & $68^{a}$ & $83^{a}$ & - & $15^{a}$ & $10^{a}$ & $20^{2}$ & $50^{2}$ & $10^{a}$ & $10^{a}$ \\
\hline
\end{tabular}

Table 10. Coefficients and $\mathrm{R}$ squares of regression equation $\mathrm{y}=\mathrm{a}+\mathrm{bx}_{1}+\mathrm{cx}_{2}$, where $\mathrm{y}$ is relative dry matter yield for the potassium rate $(s)$ (highest $=100), x_{1}$ is plant $\mathrm{N} / \mathrm{K}$-ratio or K-AAA $(\mathrm{mg} / 1)$ in surface soil, and $\mathrm{x}_{2}$ is plant

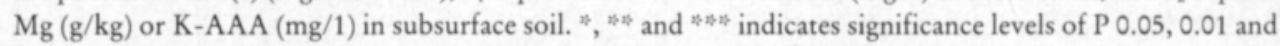
0.001 . Blanks and missing rates means lacking of significant dependences.

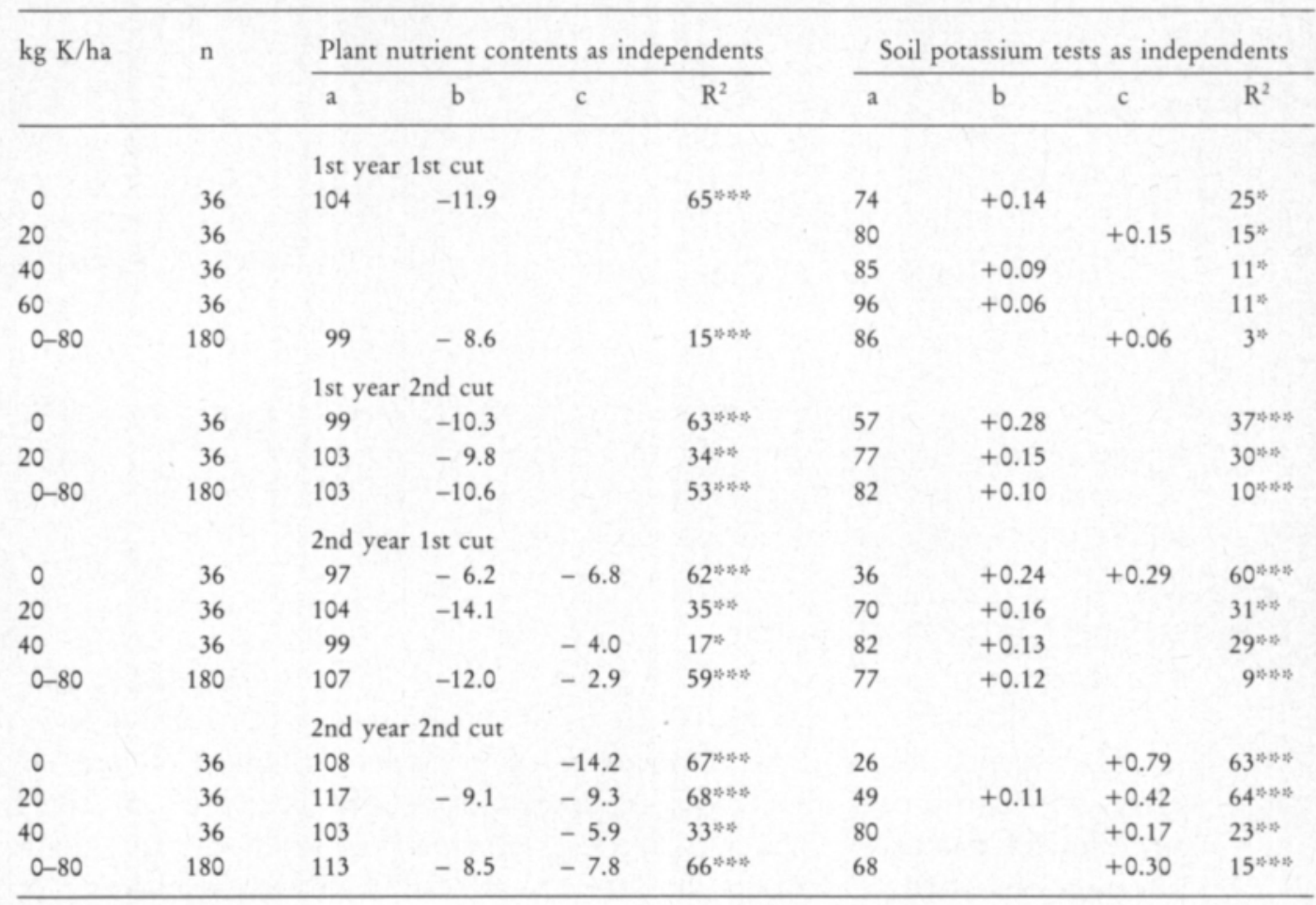


was low, 2440 feed units per hectare (SALONEN and TAINIO 1961). The steep fall of yields without adequate potassium applications and the increases of responses have been observed also in Norway (BAERUG 1977 a, HERNES 1978) and in a few trials in Finland (HEIKKILÄ and JUOLA 1976).

On soils belonging to the coarse mineral soils group, the yield increases of ley crops obtained with potassium fertilization have been variable also in previous studies (SALONEN and TAINIO 1961). On two finesand soils, no response has been observed in the first three years (SAARELA et al. 1981).

The organogenic soils, where potassium fertilization in ley cropping is most important, are not uncommon as ley soils in Finland. As calculated according to the areas of field crops within the agricultural centers (ANON. 1982 ) and the respective soil type proportions (KURKI 1982), the area of organogenic grassland soils is about 250000 hectares or $27 \%$ of the total grassland area. The mineral soils in grassland-dominated parts of Finland are coarse-textured. No less than $62 \%$ of the grassland is coarse mineral soil and only $11 \%$ clay.

The mean K-AAA value for arable Finnish peat soils is, according to a large material of Soil Fertility Service (KURKI 1982), only $66 \mathrm{mg} / \mathrm{l}$, the respective value for all arable soils being $148 \mathrm{mmg} / \mathrm{l}$. The poor potassium status of peats, together with a very weak potassium buffer power, is an inherent property of the soil type and can not be permanently corrected using water-soluble fertilizers. On peat soils, especially with heavy nitrogen dressings, the potassium content of grasses increases very sharply with incrasing potassium rates. When the apparent recovery in yield is nearly 100 $\%$, as it was at most of the sites, the effect on soil potassium content is necessarily small. A further increase of potassium rate would also increase soil potassium, but raises plant potassium excessively (ETTALA and KOSSILA 1979 ) and is uneconomic.

Under those conditions, where available potassium reserves of soil can not be maintained, potassium should be dressed separately for every cut in rates that are balanced with the actual requirement of plants. The potassium is then applied not for soil fertility but for plant fertilization, much in the same manner as nitrogen fertilizers. As potassium is a macro nutrient the required amounts of potassium are quite large, of the same order of magnitude as applied amounts of nitrogen.

On fully exhausted soil, no crop plant following the ley grass would thrive without an adequate application of potassium. The depletion of soil as a result of a negative potassium balance ought to be borne in mind also in cases where the yield response has been small or even absent. The negative residual effect of potassium uptake of ley grasses on the following crops can last several years (PENNY and WIDDOWSON 1981).

According to regression calculus, both soil and plant analyses may be useful in potassium control of leys. The rapid exhaustion of "exchangeable" soil potassium (K-AAA) in soils with a low potassium buffer power, especially peats, ought to be taken into account. Nonexchangeable potassium that is released by a strong acid seems to show the long-term potassiumreleasing ability of soil quite accurately, but further studies are necessary for 
proper evaluation of the test. Especially soils in the group of coarse mineral soils are variable in their potassium releasing abilities (KAILA 1967) and would need a kind of subclassification. Clay soils, gyttja clays exluded, are usually "rich" and organogenic soils are usually "poor" in slowly-releasing potassium.

The critical plant potassiun content (the lowest content which gives maximum yield) was highly variable in the present study. A significant share of the variation was coupled with plant nitrogen, as critical plant potassium content increased with increasing plant nitrogen content. This is not a surprising finding, but a natural consequence of anatomy and physiology of plants. Young leafy grass contains relatively more nitrogenous protoplasm than older grass with more woody supporting tissue in its stalk. Potassium not being a constituent of plant tissues but a kind of catalyst, is also needed in highest concentrations in the most active protoplasmic parts of plants.

The critical potassium content varied from under $2 \%$ to over $3 \%$. Results of other studies are mostly in agreement with this wide range (BAERUG 1977 b, MELA et al. 1977, HERNES 1978, PELTOMAA et al. 1979, TÄHTINEN 1979, SAARELA et al. 1981). As low a value as $1.6 \%$, which has been obtained by REITH et al. (1964) and quoted by ETTALA and KOSSILA (1979), may be under Finnish conditions in light of the present trials and the referred papers, a sufficient potassium content in low-proteineous hay but not in grass at the silage stage.

Acknowledgements. The field trials were planned by Professor Paavo Elonen, Head of the Department. Mrs Hilkka Tähtinen, Lic. Agr., was responsible for carrying out the trials. The soil analyses study were performed under the direction of Doctor Jouko Sippola. I wish to also thank the whole staff for its skillful work.

\section{References}

ANON. 1982. Areas of field crops in 1982. Monthly Review of Agricultural Statistics. July 1982: 216-221.

BAERUG, R. 1977 a. Nitrogen, kalium, magnesium og svovel til eng på Sør-Østlandet. I. Avlinger og jordanalyser. Summary: Nitrogen, potassium, magnesium and sulphur fertilization of forage in South-eastern Norway. I. Dry matter yield and soil analysis. Forskn. Fors. Landbr. 28: 523-548.

-1977 b. II.Kjemiske analyser av avlinger. Summary: II. Chemical analyses of the forage. Forskn. Fors. Landbr. 28: 549-547.

EGNER, H., RIEHM, H. \& DOMINGO, W. R. 1960. Untersuchungen über die chemische Bodenanalyse als Grundlage für die Beurteilung des Nährstoffzustandes der Böden. II. Chemische Extraktionsmethoden zur Phosphor- und Kaliumbestimmung. Kungl. Lantbr.högsk. Ann. 26: 199-215.

ELONEN, P. 1971. Particle-size analysis of soil. Acta Agr. Fenn. 122: 1-122.

ETTALA, E. \& KOSSILA, V. 1979. Mineral content of heavily nitrogen fertilized grass and its silage. Ann. Agric. Fenn. 18: 252-262.

HEIKKILÄ, R. \& JUOLA, P. 1977. Säilörehunurmen kalilannoitus hieta- ja metsäsaraturvemaalla. Abstract: Potassium fertilizer of grassland for silage in the sandy and fen peat soils. Suovilj.yhd. Vuosik. 81: 51-58.

HERNES, O. 1978. Stigende mengde kalium og nitrogen til eng. Summary: Increasing rates of potassium and nitrogen on meadow land. Forskn. Fors. Landbr. 29: 533-543. 
HIIVOLA, S-L., HUOKUNA, E. \& RINNE, S-L. 1974. The effect of heavy nitrogen fertilization on the quantity and quality of yields of meadow fescue and cocksfoot. Ann. Agric. Fenn. 13: 149-160.

JOY, P., LAKANEN, E. \& SILLANPÄÄ, M. 1973. Effects of heavy nitrogen dressings upon release of potassium from soils cropped with ley grasses. Ann. Agric. Fenn. 12: 172-184.

KAILA, A. 1967. Release of nonexchangeable potassium from Finnish mineral soils. J. Scient. Agric. Soc. Finl. 39: 107-118.

KERÄNEN, T. \& TAINIO, A. 1968. Hiesu- ja savimaiden kalilannoitustarpeesta. Kenttäkokeiden tuliksia vuosilta 1951-66. Zusammenfassung: Über den Kalidüngungsbedarf von Lehm- und Tonböden. Ann. Agric. Fenn. 7: 161-174.

KURKI, M. 1982. Suomen peltojen viljavuudesta III. Summary: On the fertility of Finnish tilled fields in the light of investigations of soil fertility carried out in the years 1955-1980. Viljavuuspalvelu Oy, Helsinki 1982: $181 \mathrm{p}$.

KÄHÄRI, J. \& NISSINEN, H. 1978. The mineral element contents of timothy (Pheleum pratense L.) in Finland. I. Calcium, magnesium, phosphorus, potassium, chromium, cobalt, copper, iron, manganese, sodium and zinc. Acta Agr. Scand. Suppl. 20: 26-39.

MELA, T., HAKKOLA, H. \& ÄYRÄVÄINEN, K. 1977. Typpi- ja kalilannoituksen jaoituksen vaikutus nurmen satoon ja nurmirehun laatuun. Kasvinviljelylaitoksen tiedote 6: 1-27.

PELTOMAA, R., POHJANHEIMO, O. \& HUOKUNA, E. 1979. Pintakalkituksen ja K-lannoituksen vaikutus nurmen satoon ja sen $\mathrm{N}-, \mathrm{P}-, \mathrm{K}-, \mathrm{Ca}-\mathrm{ja} \mathrm{Mg}$-pitoisuuteen. Maantutkimuslaitoksen tiedote 6: $1-24$

PENNY, A. \& WIDDOWSON, F. V. 1980. An experiment begun in 1958 measuring effects of $\mathrm{N}, \mathrm{P}$ and $\mathrm{K}$ fertilizers on yield and $\mathrm{N}, \mathrm{P}$ and $\mathrm{K}$ contents of grass. 2. Residual effects on arable crops, 1968-76. J. Agric.Sci. 95: 583-595.

REITH, J. W. S., INKSON, R. H. E., HOLMES, W., MACLUSKY, D. S., REID, D., HEDDLE, R. G. \& COPEMAN, G. K. F. 1964. The effects of fertilizers on herbage production. II. The effect of nitrogen, phosphorus and potassium on botanical and chemical composition. J. Agric. Sci. 63: 209-219.

RINNE, S-L., SILLANPÄÄ, M., HUOKUNA, E. \& HIIVOLA, S-L. 1974. Effects of heavy nitrogen fertilization on potassium, calcium, magnesium and phosphorus contents in ley grasses. Ann. Agric. Fenn. 13: 96-108.

SAARELA, I., HAKKOLA, H., LINNOMÄKI, H. \& KÖYLIJÄRVI, J. 1981. Nurmen pintakalkitus, sadetus, typpi- ja kaliumlannoitus. Monitekijäkokeiden tuloksia. Maanviljelyskemian ja -fysiikan laitoksen tiedote 15: 1-37.

SALONEN, M. \& TAINIO, A. 1961. Kalilannoitusta koskevia tutkimuksia. Summary: Investigations on potash fertilization. Publ. Finn. State Agric. Res. Board 185: 1-60.

SILLANPÄÄ, M. \& RINNE, S-L. 1975. The effect of heavy nitrogen fertilization on the uptake of nutrients and on some properties of soils cropped with grasses. Ann. Agric. Fenn. 14: 210-226.

TARES, T. and SIPPOLA, J. 1978. Changes in pH, in eloctrical conductibity and in the extractable amounts of mineral elements in soil, and the utilization and losses of the elements in some field experiments. Acta Agr. Scand. Suppl. 20: 90-113.

TÄHTINEN, H. 1979. The effect of nitrogen fertilizer on the potassium requirement of grassland for silage. Ann. Agric. Fenn., 18: 231-245.

VUORINEN, J. \& MÄKITIE, O. 1955. The method of soil testing in use in Finland. Agrogeol. Publ. 63: $1-44$.

Ms received April 29, 1983 


\section{Kaliummäärän vaikutus timotein satoon}

\section{Into Saarela}

Maatalouden tutkimuskeskus, Maanviljelyskemian ja -fysiikan osasto, 31600 Jokioinen

Viittä kaliumlannoitustasoa (0-80 kg K/ha/niitto) verrattiin yhdeksällä koepaikalla timoteinurmilla, jotka lannoitettiin ja niitettiin kaksi kerta vuodessa.

Neljällä turvemaalla kahden vuoden keskisato oli ilman kaliumlannoitusta 34-66 \% riittävällä kaliumlannoituksella saadusta sadosta. Yhdellä multamaalla ilman kaliumlannoitusta saatu suhteellinen sato oli $81 \%$ ja yhdellä karkeahietamaalla $76 \%$. Kahdella runsasmultaisella hietamoreenimaalla sadonlisäys oli $5 \%$. Yhdellä hiesusavimaalla ei saatu merkitsevää sadonlisäystä.

Kaliumlannoituksen vaikutus suureni kokeen aikana maan kaliumvarojen ehtymisen mukaisesti. Kuudella koepaikalla, joilla kaliumlannoitus eniten lisäsi satoa, ilman kaliumlannoitusta saadut suhteelliset sadot olivat neljässä ensimmäisessä niitossa keskimäärin $88,75,58$ ja $45 \%$. Suurinta satoa varten kaliumía tarvittiin eloperäisillä mailla ja karkeahietamaalla niittoa kohti $60-80 \mathrm{~kg} / \mathrm{ha}$, kolmella muulla kivennäismaalla riitti $20 \mathrm{~kg} / \mathrm{ha} /$ niitto.

Kaliumlannoitus kohotti jyrkästi sadon kaliumpitoisuutta ja alensi sadon typpi-, kalsiumja magnesiumpitoisuutta. Voimakkaassa kaliumin puutteessa heinän magnesiumpitoisuus nousi epätavallisen korkeaksi. Kokeiden lopussa maat olivat melko tyhjiä kasveille käyttökelpoisesta kaliumista. Jankosta $20-40 \mathrm{~cm}: n$ syvyydestä kalium oli käytetty tarkemmin kuin kyntökerroksesta.

Alhaisin suurimman sadon tuottava sadon kaliumpitoisuus vaihteli alle kahdesta yli kolmeen prosenttiin kasvin kuiva-aineesta. Sadon kalium/typpisuhde oli tarkempi kaliumin riittävyyden osoittaja kuin kaliumpitoisuus. Kaliumin puute pienensi satoa kasvin $\mathrm{K} / \mathrm{N}$ suhteen jäädessä alle yhden. 